\title{
One-Step Synthesis and Photoluminescence Eva- luation of Cadmium-containing Quantum Dots
}

\author{
Nisha Shukla ${ }^{1, *}, \quad$ Michael M. Nigra ${ }^{2}, \quad$ Abigail D. Ondeck ${ }^{2}$
}

(Received 8 November 2011; accepted 22 March 2012; publication online 28 March 2012.)

\begin{abstract}
We have developed a simple one-step process for synthesis of ternary quantum dots (ZnCdSe, $\mathrm{MgCdSe}$ ) with photoluminescence wavelengths ranging from the red to the blue region of the visible spectrum. The primary aim of this work was to develop a synthesis for the preparation of Cd-containing quantum dots using a Cd precursor with lower toxicity than those used in common syntheses. This synthesis makes use of $\mathrm{Cd}(\mathrm{acac})_{2}$ which is significantly less toxic than precursors such as $\mathrm{CdO}$ and $\mathrm{CdCl}_{2}$. We have studied the effect of solvent boiling point, precursors and reaction time on the photoluminescence properties of the ternary quantum dots. Ternary quantum dots synthesized from $\mathrm{Cd}(\mathrm{acac})_{2}$ in low boiling point solvents have photoluminescence wavelengths in the blue region, while those synthesized in high boiling point solvents have photoluminescence wavelengths in the red region.
\end{abstract}

Keywords: Quantum dots; ZnCdSe; MgCdSe; Photoluminescence

Citation: Nisha Shukla, Michael M. Nigra and Abigail D. Ondeck, "One-Step Synthesis and Photoluminescence Evaluation of Cadmium-Containing Quantum Dots", Nano-Micro Lett. 4 (1), 52-56 (2012). http://dx. doi.org/10.3786/nml.v4i1.p52-56

\section{Introduction}

Quantum dots are small semiconductor nanoparticles with electron energy levels that are discrete rather than continuous. The size of the semiconductor nanoparticles determines the spacing of their energy levels and thus, their band gap. The band gap increases as the quantum dots size decreases and thus, the band gaps of quantum dots are tunable. As a result of their high band gaps, smaller quantum dots absorb light at shorter wavelengths than larger quantum dots. The other parameter which controls the band gap of quantum dots is their composition. By controlling both the quantum dot size and composition, it is possible to create quantum dots that absorb light at frequencies spanning the entire solar spectrum. As a result, these materials have great potential for use in photovoltaic applications [1-3].

There are several reports of the preparation of ternary quantum dots using wet chemical syntheses [19]. Most of these syntheses are two-step processes.
Knoll et al. [4] reported the synthesis of ZnCdSe quantum dots using a two-step process. In a two-step synthesis process CdSe quantum dots were synthesized first and then used as seeds to make core-shell structures of ZnSe with CdSe inside and ZnSe outside. In this type of two-step process both CdSe and ZnSe are synthesized separately and there no alloy formation of CdSe and ZnSe until they are annealed. The core-shell structures of CdSe core and $\mathrm{ZnSe}$ shell was then heated to form alloy $\mathrm{ZnCdSe}$ quantum dots. In that process [4], it was observed that changing the amount of $\mathrm{Zn}$ caused a shift in the photoluminescence peak wavelength. The quantum dots of pure CdSe $(0 \% \mathrm{Zn})$ had photoluminescence peaks in the red region of the solar spectrum while photoluminescence peaks in the blue region of the solar spectrum were observed for $\mathrm{Zn}_{0.67} \mathrm{Cd}_{0.33}$ Se quantum dots. In similar work, other types of three and four component quantum dots have been synthesized using two-step processes that generate core-shell structures [10-12]. The component combinations previously re-

\footnotetext{
${ }^{1}$ Institute for Complex Engineered Systems, Carnegie Mellon University, Pittsburgh, PA 15213, U.S.A.

${ }^{2}$ Department of Chemical Engineering, Carnegie Mellon University, Pittsburgh, PA 15213, U.S.A.

*Corresponding author. E-mail: nisha@andrew.cmu.edu
} 
ported include: CdSe/ZnS, CdSe/ZnSe, CdS/ZnS and CdSe/CdS [10-15]. The core-shell structures are then heated to form alloys. Needless to say, from the point of view of large scale preparation of quantum dots, a one step process would be superior to a two step process. In our method of synthesis we have used one-step synthesis as explained in the experimental section. We have used cadmium and zinc precursors at the same time in the reaction mixture in the presence of selenium and reduced these precursors simultaneously.

A second issue with the reported syntheses of these types of quantum dots is that many of the precursors are highly toxic, which presents another problem in the scale-up of the preparation methods. Typical $\mathrm{Cd}$ containing precursors such as $\mathrm{CdO}, \mathrm{Cd}\left(\mathrm{CH}_{3}\right)_{2}, \mathrm{CdSO}_{4}$, $\mathrm{Cd}\left(\mathrm{C}_{17} \mathrm{H}_{35} \mathrm{COO}\right)_{2}$ all have Hazardous Materials Identification System (HMIS) and National Fire Protection Association (NFPA) ratings of 4 . Chemicals with ratings of 3 and 4 are extremely toxic or hazardous materials. $\mathrm{CdCl}_{2}$ and $\mathrm{Cd}\left(\mathrm{NO}_{3}\right)_{2}$ have NFPA and HMIS ratings of 3 . Another $\mathrm{Cd}$ precursor reported in literature is $\mathrm{Cd}\left(\mathrm{CH}_{3} \mathrm{COO}\right)_{2}$ with NFPA and HMIS ratings of 2 (moderately toxic or hazardous). The HMIS and NFPA health ratings for $\mathrm{Zn}(\mathrm{acac})_{2}$ are 2 . Again, scale-up of processes for preparation of quantum dots composed of these elements would benefit from the development of syntheses using less toxic and less dangerous precursors.

The objective of the work reported has been to demonstrate a one-step synthesis of ternary quantum dots using low toxicity chemicals and to understand the synthesis parameters that influence the properties of the resulting ternary quantum dots. This understanding will be used to develop ternary quantum dots with improved photoluminescence properties. The ultimate goal is to prepare ternary quantum dots with narrow absorption peaks at any wavelength. In this work we have used two types of solvents $\left(1,2\right.$-dichlorobenzene, b.p. $180^{\circ} \mathrm{C}$; 1 -octadecene, b.p. $\left.315^{\circ} \mathrm{C}\right)$, and a variety of $\mathrm{Cd}$ precursors $\left(\mathrm{CdO}, \mathrm{CdCl}_{2}\right.$ and $\left.\mathrm{Cd}(\mathrm{acac})_{2}\right)$ and $\mathrm{M}(\mathrm{acac})_{2}$ precursors $(\mathrm{M}=\mathrm{Zn}, \mathrm{Mg})$ to synthesize ternary alloy quantum dots. The type of Cd precursors did not have any impact on the photoluminescence peaks of the chemically synthesized quantum dots. As a result, we have reported results only from quantum dots prepared using $\mathrm{Cd}(\mathrm{acac})_{2}$ as it is the least hazardous of the three with a HMIS health rating of 1 and a NFPA health rating of 1 (slightly toxic or hazardous material). In this work, several types of ternary quantum dots including $\mathrm{ZnCdSe}$ and $\mathrm{MgCdSe}$ were synthesized and the effect of solvent on the photoluminescence properties of these quantum dots was studied. We have focused on the photoluminescence properties of these quantum dots prepared using a one-step process starting with low toxicity precursors. Ternary quantum dots synthesized in low boiling point solvents have photoluminescence wavelengths in the blue region, while those synthesized in high boiling point solvents have photoluminescence wavelengths in the red region.

\section{Experimental}

ZnCdSe quantum dots were synthesized using a one-step process involving reduction of $\mathrm{Cd}(\mathrm{acac})_{2}$ and $\mathrm{Zn}(\mathrm{acac})_{2}$ in a low boiling point polar solvent (1,2dichlorobenzene, b.p. $180^{\circ} \mathrm{C}$ ) and in the presence of surfactants (oleic acid). The synthesis of ternary alloy quantum dots was conducted in a moisture and oxygen free atmosphere. The $\mathrm{Cd}(\mathrm{acac})_{2}(0.15 \mathrm{mmol})$ and $\mathrm{Zn}(\mathrm{acac})_{2}(0.06 \mathrm{mmol})$ were heated in the $1,2-$ dichlorobenzene $(10 \mathrm{ml})$ in the presence of oleic acid $(0.6 \mathrm{ml})$ until they dissolved. Then a $1 \mathrm{ml}$ solution of Se in trioctylphosphine (TOP) in 1,2-dichlorobenzene (concentration of $0.07 \mathrm{mmol} / \mathrm{ml}$ ) was added to the reaction mixture (details are in the results section). The reaction mixture was allowed to reflux $\left(180^{\circ} \mathrm{C}\right)$ and 0.5 $\mathrm{ml}$ aliquots of the reaction mixture were withdrawn after different intervals of time. The aliquots were diluted in $3 \mathrm{ml}$ of toluene in order to obtain photoluminescence spectra. This diluted solution in toluene still had some dichlorobenzene. Similar experiments were also performed using a high boiling point solvent 1-octadecene (b.p. $315^{\circ} \mathrm{C}$ ). In addition to synthesis of $\mathrm{ZnCdSe}$, we have extended our synthesis method to prepare $\mathrm{MgCdSe}$ using $\mathrm{Mg}(\mathrm{acac})_{2}$ as a precursor.

The photoluminescence spectra were taken using a PTI Timemaster series spectrofluorometer fitted with a PMT sensor. The excitation source was a Xe lamp emitting at $350 \mathrm{~nm}$. The scan range chosen for each sample was $375-650 \mathrm{~nm}$ and spectra were obtained using a $1 \mathrm{~nm}$ step and a $0.5 \mathrm{sec}$ integration time. Spectra were captured using the FeliX32 ${ }^{\mathrm{TM}}$ Advanced Fluorescence Analysis Software Package. In our experiments the intensity of photoluminescence peaks is reflective of the concentration of particles which is not controlled in our experiments.

All the solvents and chemicals $\left(\mathrm{Cd}(\mathrm{acac})_{2}(99 \%)\right.$, $\mathrm{Zn}(\mathrm{acac})_{2}, \mathrm{Mg}(\mathrm{acac})_{2}$, Se powder, hexane, ethanol, 1,2-dichlorobenzene, octadecene, oleic acid, trioctylphoshine, toluene and dioctylether) used for the synthesis and washing of the ZnCdSe, MgCdSe quantum dots were obtained from Aldrich Chemical Co. and were used as obtained without further purification. Ethanol was obtained in Sureseal ${ }^{\mathrm{TM}}$ bottles with very low water content $(<0.05 \%)$. The hexane was $99 \%$ pure with low water content.

\section{Results}

There are a number of parameters that influence the synthesis and final properties of ternary quantum dots. This work focuses on the effects of using $\mathrm{M}(\mathrm{acac})_{2}$ (M 
$=\mathrm{Cd}, \mathrm{Zn}, \mathrm{Mg}$ ) precursors in the synthesis of ternary quantum dots. The other variable which was explored in the synthesis of ternary alloy quantum dots was the type of solvent used in the synthesis. Solvents with different boiling points and polarity can influence the synthesis of ternary alloy quantum dots. Figure 1 shows the photoluminescence spectra of $\mathrm{ZnCdSe}$ quantum dots which were synthesized using 1,2-dichlorbenzene as a solvent. The chemical precursors used in this experiment were $\mathrm{Zn}(\mathrm{acac})_{2}(0.06 \mathrm{mmol})$ and $\mathrm{Cd}(\mathrm{acac})_{2}(0.15$ mmol) combined in the $10 \mathrm{ml}$ of 1,2-dichlorobenzene. The molar ratio of Cd:Se was 1.8. The molar ratio of $\mathrm{Zn}: \mathrm{Cd}=0.4$ was chosen. We wanted to gradually increase the amount of $\mathrm{Zn}$ precursor to study the affect of $\mathrm{Zn}$ concentration on the photoluminescence properties of the $\mathrm{ZnCdSe}$ quantum dots. Increasing the $\mathrm{Zn}$ concentration is expected to result in a blue shift of the photoluminescence peak [4]. After heating the reaction mixture of $\mathrm{Zn}(\mathrm{acac})_{2}$ and $\mathrm{Cd}(\mathrm{acac})_{2}$ in $10 \mathrm{ml}$ of 1,2-dichlorobenzene to the boiling point of $180^{\circ} \mathrm{C}$, a $1 \mathrm{ml}$ solution of Se powder in trioctylphosphine (TOP) and 1,2-dichlorobenzene was added to the reaction mixture (concentration of $0.07 \mathrm{mmol} / \mathrm{ml}$ ). The reaction mixture was heated and aliquots $(0.5 \mathrm{ml})$ were withdrawn after $5 \mathrm{~min}, 10 \mathrm{~min}, 15 \mathrm{~min}$ and $2 \mathrm{~h}$. The aliquots of the reaction mixture $(0.1 \mathrm{ml})$ were diluted in toluene $(2.5 \mathrm{ml})$ before taking photoluminescence measurements. As shown in Fig. 1 (in dotted lines), these CdZnSe quantum dots have narrow photoluminescence peaks (FWHM $\sim 30-35 \mathrm{~nm}$ ) in the blue to yellow region (494-580 nm). The photoluminescence wavelengths increase with reaction time. This shift in the photoluminescence peaks is caused by the fact that quantum dots grow in size as the reaction proceeds. The photoluminescence spectra of $\mathrm{ZnCdSe}$ quantum dots synthesized in 1,2-dichlorobenzene with a $\mathrm{Zn}: \mathrm{Cd}$ precursor molar ratio of 1.2 is shown in solid line in Fig. 1. The photoluminescence spectra show wavelengths in the range 483-554 $\mathrm{nm}$. The increased $\mathrm{Zn}$ content results in a net blue shift of the photoluminescence peaks. This is consistent with a prior report that increasing the Zn content of ZnCdSe quantum dots causes them to absorb at shorter wavelengths [4]. In that work, with increasing the mole fraction of $\mathrm{Zn}$ from 0 , to $0.28,0.44,0.55$ and 0.67 the photoluminescence peak shifted from $630 \mathrm{~nm}$ to $490 \mathrm{~nm}$. The band gap of CdSe is $1.84 \mathrm{eV}$ while that of $\mathrm{ZnSe}$ is $2.82 \mathrm{eV}$ which is consistent with this shift. The molar ratio of $\mathrm{Cd}$ :Se in the experiment shown in the Fig. 2 was 1.4. Our previous studies [15] have shown that in the absence of the $\mathrm{Zn}$ precursor, with a similar molar ratio of $\mathrm{Cd}$ :Se, the photoluminescence peaks were observed at $503 \mathrm{~nm}$ (after 4-5 min of reaction time) for CdSe quantum dots. The wavelength of the photoluminescence peak for CdSe quantum dots is almost $20 \mathrm{~nm}$ longer than that observed for CdZnSe quantum dots as shown in Fig. 1 (solid lines) (see details in Table 1).

Our next goal was to study the effect of synthesis solvent on the photoluminescence of ternary quantum dots. Figure 2 shows the photoluminescence spectra of ZnCdSe synthesized with a $\mathrm{Zn}: \mathrm{Cd}$ molar ratio of 0.4 and $\mathrm{Cd}$ :Se molar ratio of 1.4 (this ratio of $\mathrm{Zn}: \mathrm{Cd}$ and $\mathrm{Cd}: \mathrm{Se}$ is close to that of the experiment shown in Fig. 1) in the reaction mixture but using 1-octadecene as the solvent rather than 1,2-dichlorobenzene. One of the principal differences between these two solvents is that the boiling point of 1 -octadecene is $315^{\circ} \mathrm{C}$, whereas, the boiling point of 1,2 -dichlorobenzene is $180^{\circ} \mathrm{C}$. Figure 2 shows that photoluminescence of the quantum dots synthesized at $315^{\circ} \mathrm{C}$ in 1-octadecene occurs at $\sim 600 \mathrm{~nm}$ and there is no shift in the photoluminescence peak wavelength as the reaction proceeds. After 5 min of reaction time in 1-octadecene the photoluminescence peak wavelength is $600 \mathrm{~nm}$, whereas, in 1,2-dichlorobenzene the photoluminescence peak wavelength was $494 \mathrm{~nm}$ after $5 \mathrm{~min}$ (Fig. 1). This observation suggests that the reaction in 1-octadecene reached completion rather quickly and produced relatively large quantum dots. Another point to note is that the photoluminescence peaks of $\mathrm{ZnCdSe}$ quantum dots synthesized in 1-octadecene are broader (FWHM $\sim 50 \mathrm{~nm}$ ) than those of the ZnCdSe quantum dots synthesized in 1,2-dichlorobenzene (FWHM $\sim 30-35 \mathrm{~nm}$ ). This suggests that the synthesis at higher temperature yields as a broader distribution of sizes.

One advantage of this one-step synthesis is that it can be performed with numerous types of $\mathrm{M}(\text { acac })_{2}$ precursors ( $\mathrm{M}$ denotes any metal). Our next experiment attempted to synthesize MgCdSe quantum dots using $0.084 \mathrm{mmol} \mathrm{Mg}(\mathrm{acac})_{2}$ and $0.20 \mathrm{mmol} \mathrm{Cd}(\mathrm{acac})_{2}$ $\mathrm{Mg}: \mathrm{Cd}$ precursors $=0.4$ and $\mathrm{Cd}: \mathrm{Se}=2.8)$ in 1,2 dichlorobenzene. The band gap of MgSe is $5.4 \mathrm{eV}$ which is higher than that of ZnSe $(2.82 \mathrm{eV})$. As result, substitution of $\mathrm{Mg}$ for $\mathrm{Zn}$ ought to produce quantum dots with higher band gaps and result in a blue shift of the photoluminescence peaks. The photoluminescence spectra shown in Fig. 3 show that after $5 \mathrm{~min}$ of

Table 1 Photoluminescence peak maxima of CdSe and ZnCdSe quantum dots synthesized in 1, 2 dichlorobenzene (DCB).

\begin{tabular}{|c|c|c|c|c|c|c|c|c|c|c|}
\hline Cd Precursor & $\mathrm{Cd} / \mathrm{Se}$ & solvent & b. $\mathrm{p}^{\circ} \mathrm{C}$ & $\mathrm{Zn} / \mathrm{Cd}$ & $2 \min$ & $4 \min$ & $10 \mathrm{~min}$ & $15 \mathrm{~min}$ & $20 \mathrm{~min}$ & $2 \mathrm{~h}$ \\
\hline $\mathrm{Cd}(\mathrm{acac})_{2}$ & 1.3 & $\mathrm{DCB}$ & 180 & 0 & 493 & 503 & & 506 & 510 & \\
\hline $\mathrm{Cd}(\mathrm{acac})_{2}$ & 1.4 & DCB & 180 & 1.2 & & 483 & 493 & & 505 & 554 \\
\hline
\end{tabular}




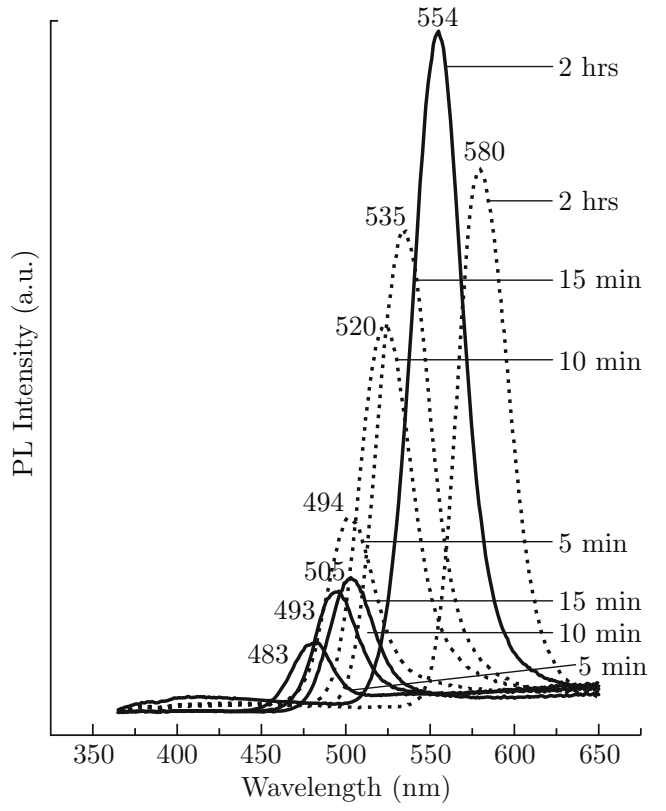

Fig. 1 Photoluminescence spectra of ZnCdSe quantum dots synthesized in 1,2-dichlorobenzene (b.p. $180^{\circ} \mathrm{C}$ ) for various reaction times and various molar ratio of $\mathrm{Zn}$ :Cd precursors used in the synthesis. Photoluminescence peaks shown in dotted lines correspond to $\mathrm{Zn}$ :Cd precursor ratio of 0.4 . Photoluminescence peaks shown in solid lines correspond to $\mathrm{Zn}: \mathrm{Cd}$ precursor ratio of 1.2 . The photoluminescence peaks for quantum dots synthesized using a molar ratio of $\mathrm{Zn}: \mathrm{Cd}=1.2$ occur at shorter wavelengths than for those synthesized using a molar ratio of $\mathrm{Zn}: \mathrm{Cd}=0.4$.

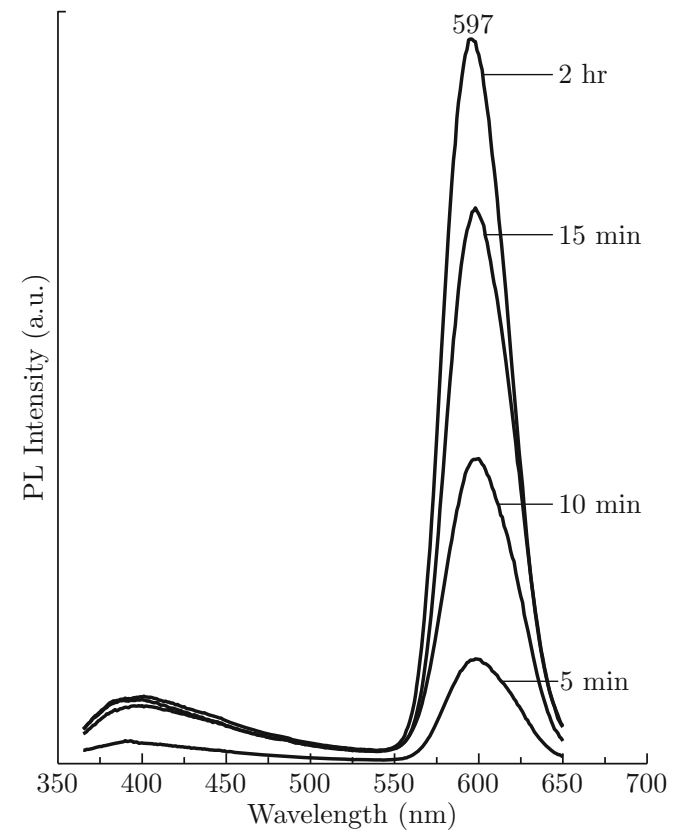

Fig. 2 Photoluminescence spectra of $\mathrm{ZnCdSe}$ quantum dots synthesized in 1-octadecence (b.p. $315^{\circ} \mathrm{C}$ ) for various reaction times. The molar ratio of $\mathrm{Zn}$ :Cd precursors used in the synthesis was 0.4 . The higher reaction temperature results in larger particles than those synthesized in 1,2-dichlorobenzene and photoluminescence at longer wavelengths. reaction time the photoluminescence peak for the $\mathrm{MgCdSe}$ quantum dots is at $468 \mathrm{~nm}$, whereas, after a similar reaction time the photoluminescence peak for ZnCdSe quantum dots was observed at 494 nm (Fig. 1). The photoluminescence peaks for the $\mathrm{MgCdSe}$ quantum dots obtained after 10-15 min of reaction were also observed at shorter wavelengths than those for $\mathrm{ZnCdSe}$ quantum dots synthesized for similar periods of time. The results of the synthesis of MgCdSe quantum dots in 1-octadecene were similar to those observed in the case $\mathrm{ZnCdSe}$ quantum dots as shown in Fig. 2. The photoluminescence peaks for $\mathrm{MgCdSe}$ quantum dots synthesized in 1-octadecene were all located at $630 \mathrm{~nm}$ and were quite broad. The high reaction temperature presumably resulted in the synthesis of large quantum dots. This clearly shows that our synthesis method is capable of using $\mathrm{Mg}(\mathrm{acac})_{2}$ for generating quantum dots to photoluminescence in the blue region.

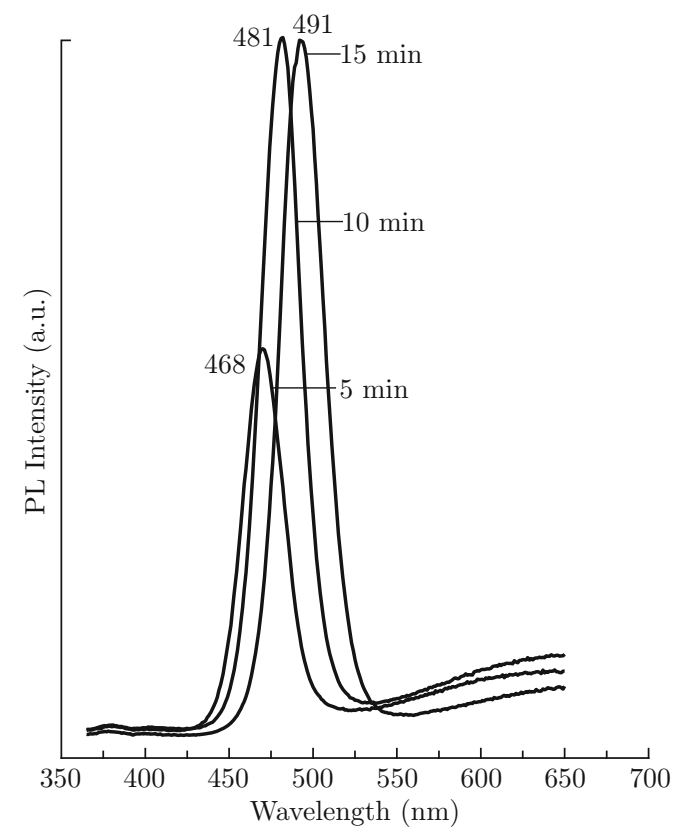

Fig. 3 Photoluminescence spectra of MgCdSe quantum dots synthesized in 1,2-dichlorobenzene for various reaction times. The molar ratio of $\mathrm{Mg}$ : $\mathrm{Cd}$ precursors used in the synthesis is 0.4 . The substitution of $\mathrm{Mg}$ for $\mathrm{Zn}$ results in a shift of photoluminescence peaks towards blue region.

\section{Conclusions}

This work demonstrates a one-step synthesis of ternary quantum dots. This synthesis method allows the use of various $\mathrm{M}(\mathrm{acac})_{2}(\mathrm{M}=\mathrm{Cd}, \mathrm{Mg}, \mathrm{Zn})$ precursors which are less hazardous than many precursors used in similar syntheses of ternary alloy quantum dots. The boiling point of the solvent influences the reaction; the higher the reaction temperature the longer the wavelength at which quantum dots photoluminescence. In addition, the molar ratio of the precursors also dic- 
tates the photoluminescence properties. Furthermore, quantum dots with elements that decrease the bandgap of the bulk semiconductor increase the photoluminescence wavelength of the quantum dots.

\section{Acknowledgments:}

The authors would like to thank CIT (Carnegie Institute of Technology), and ICES (Institute of Complex Engineered System) for the financial support of this work.

\section{References}

[1] W. U. Huynh, J. J. Dittmer and A. P. Alivisatos, Science 295, 2425 (2002). http://dx.doi.org/10.1126/ science. 1069156

[2] W. U. Huynh, X. Peng and A. P. Alivisatos, Adv. Mater. 11, 923 (1999). http://dx.doi.org/ 10.1002/(SICI) 1521-4095(199908) 11: 11<923: : AID-ADMA923>3.0.CD;2-T

[3] D. S. Ginger, N. Greenham, X. Peng and A. P. Alivisatos, Synth. Met. 84, 545 (1997). http://dx. doi .org/10.1016/S0379-6779(97)80852-1

[4] X. Zhong, M. Han, Z. Dong, T. J. White and W. Knoll, J. Am. Chem. Soc. 125, 8589 (2003). http://dx.doi. org/10.1021/ja035096m

[5] X. Zhong, Y. Feng, W. Knoll and M. Han, J. Am. Chem. Soc. 125, 13559 (2003). http://dx.doi.org/ 10.1021/ja036683a
[6] P. M. Allen and M. G. Bawendi, J. Am. Chem. Soc. 130, 9240 (2008). http://dx.doi.org/10.1021/ ja8036349

[7] J. H. Bang, W. H. Suh and K. S. Suslick, Chem. Mater. 20, 4033 (2008). http://dx.doi.org/10.1021/ cm800453t

[8] K. Nose, T. Omata and S. O-Yao-Matsuo, J. Phys. Chem. C. 113, 3455 (2009). http://dx.doi.org/10. 1021/jp809398k

[9] O. I. Micic and A. J. Nozik, J. Lumines 70, 95 (1996).

[10] B. O. Dabbousi, J. Rodriguez-Viejo, F. V. Milulec, J. R. Heine, H. Mattoussi, R. Ober, K. F. Jensen and M. G. Bawendi, J. Phys. Chem. 101, 9463 (1997). http: // dx.doi.org/10.1021/jp971091y

[11] P. Reiss, J. Bleuse and A. Pron, Nanolett. 2, 781 (2002). http://dx.doi.org/10.1021/n1025596y

[12] J. S. Steckel, J. P. Zimmer, S. Coe-Sulliavan, N. E. Scott, V. Bulovic and M. G. Bawendi, Angew. Chem. Int. Ed. 43, 2154 (2004). http://dx.doi.org/ 10.1002/anie. 200453728

[13] X. Peng, M. C. Schlamp, A. V. Kadavanich and A. P. Alivisatos, J. Am. Chem. Soc. 119, 7019 (1997). http://dx.doi.org/10.1021/ja970754m

[14] J. S. Steckel, J. P. Zimmer, S. Coe-Sullivan, N. E. Stott, V. Bulovi and M. G. Bawendi, Angew Chem. 43, 2154 (2004). http://dx.doi.org/10.1002/anie. 200453728

[15] N. Shukla and M. M. Nigra, Luminescence 25, 14 (2010). 